\title{
WHEN SYNTACTIC ERRORS GO UNNOTICED: AN AMRI STUDY OF THE EFFECT OF SEMANTICS ON SYNTAX
}

Sharlene D. Newman ${ }^{1}$ Indiana University, Bloomington

Ben Pruce Indiana University, Bloomington

Toshikazu Ikuta Indiana University, Bloomington

Thomas Burns, Jr. Indiana University, Bloomington

\begin{abstract}
In our day to day conversations there are often times when we fail to notice syntactic errors. But why? In this study we conducted both a behavioral and an fMRI study to address this question. The results showed that participants were more likely to fail to detect a morphosyntactic violation if the sentence constituents were semantically related to each other than if they were unrelated. In addition, the related anomalous sentences elicited stronger activation of the left inferior frontal gyrus. Three separate clusters of activation were observed encompassing BA $44, \mathrm{BA} 45 / 46$ and one at the junction of the inferior frontal and precentral sulci. While previous work has demonstrated that
\end{abstract}

\begin{tabular}{|l|l|l|l|l|}
\hline Ilha do Desterro & Florianópolis & $n^{\circ} 63$ & p. 015-035 & jul/dez 2012 \\
\hline
\end{tabular}


semantics information such as plausibility and world knowledge does not have a significant impact on comprehension, it does affect anomaly detection. One theory of language processing that fit the results is "good enough" theory which suggests that we fail to generate a complete representation of the input, particularly when the input describes plausible and/or familiar events.

Keywords: fMRI, syntax, semantics

\section{Introduction}

During normal communication sentence constituents are related to each other and as a result allow for the use of prior knowledge to aid in comprehension. For example, when discussing an event that occurred in a hospital it would be odd to encounter the word elephant but not so odd to encounter the words doctor or nurse. The use of prior knowledge in comprehension may be expected to assist processing and may have an impact on syntactic level processing.

While the influence of sentence structure on meaning has been well documented, the characterization of the effects of semantics on syntactic processing has been more elusive. There is one ERP effect that demonstrates that semantics can have a significant impact on syntax, the semantic P600 - the P600 has been observed during the processing of syntactic violations (Hagoort et al., 1993; Kuperberg et al., 2005). Typically the semantic P600 has been observed in studies that examined animacy violations (Hoeks et al., 2004; Kim et al., 2005). While semantic features such as animacy have been found to have an impact on syntactic processing, other semantic/world knowledge information has failed to show a significant impact on syntax (Bornkessel-Schlesewsky \& Schlesewsky, 2008; Newman \& Ikuta, 2010). 
The extended ArgumentDependency Model(eADM; BornkesselSchlesewsky \& Schlesewsky, 2008) suggests that semantic features like animacy (which is referred to as prominence information) is processed independently and parallel to world knowledge/semantic relatedness (referred to as plausibility information). Additionally, the model suggests that prominence information is processed earlier and can have an immediate impact on sentence level processing while plausibility information does not have an impact on sentence level, syntactic processing (see Bornkessel-Schlesewsky \& Schlesewsky, 2008 for details). In fact, we found support for this claim in a study that examined comprehension (Newman \& Ikuta, 2010). In that study the impact of the semantic relatedness of the sentence constituents on the comprehension of conjoined active and object related sentences was examined. A region of the left inferior frontal gyrus (LIFG), BA 44, which has been strongly associated with syntactic processing (Friedercici et al., 2003; Lee \& Newman, 2010; Newman et al., 2003; Ni et al., 2000) failed to show an interaction between syntactic complexity and semantic relatedness and only revealed complexity effects. This result was interpreted as support for the eADM hypothesis that plausibility has little impact on syntactic analysis, at least during a comprehension task.

While the above model assumes a complete, algorithmic analysis of both syntax and semantics during comprehension, an alternative theory suggests that semantic and syntactic representations created by the comprehender are "good enough" to satisfy the comprehender's needs (Ferreira et al., 2002, 2007; Sanford \& Sturt, 2002), remembering that the comprehender's needs are typically to understand/comprehend the intended message. Therefore, no complete representation of the sentence is necessarily generated, or 
as described by Sanford, the representation is underspecified. Two pieces of evidence have been presented that bring into question the idea that comprehenders create a complete representation: 1) people typically have a shallow understanding of the meaning of a sentence; and 2) people sometimes completely misunderstand sentences. The "good enough" processing idea suggests that general knowledge does indeed play a significant role in sentence level processing in that it encourages this superficial sentence processing. One example is the verbal illusion or depth-charge sentences (Sanford \& Sturt, 2002; Wason \& Reich, 1979); for example, No head injury is too trivial to be ignored. This sentence is typically interpreted as "A head injury should never be ignored no matter how trivial." This interpretation is guided by our knowledge of how damaging a head injury can be. However, grammatically the sentence should be interpreted as "Regardless of how trivial the head injury is it should be ignored."

The current study contributes to the debate regarding the influence of semantic information on sentence level processing, specifically syntactic level processing. Here we examined the effect of the semantic relatedness of sentence constituents on the ability to detect morphosyntactic (subject/verb agreement) violations. While semantic relatedness has not been found to affect syntax during comprehension, an effect may be observed during anomaly detection. If we assume "good enough" processing is taking place in order to have fast comprehension and that this processing relies on heuristics and regularities like the semantic relationships or prior experience with the concepts within the sentence, then sentences that contain semantically related constitutes should have facilitated processing, during a comprehension task. However, that may not be the case when the task is not comprehension but is instead the detection of a morphosyntactic violation. This is because morphosyntax has little 
impact on the overall meaning of the sentence and therefore may not be processed fully under "good enough" processing.

The focus of the current study is the left inferior frontal gyrus (LIFG). Although the IFG is oftentimes discussed as though it is one unitary, homogenous region, it is actually composed of distinct areas which are segregated by anatomical landmarks and cytoarchitectural differences. Recent studies have attributed distinct sub-regions of the LIFG to different sentence level processes. For example, BA 44 in particular has been strongly linked to syntax and has been shown to be involved in processing prominence information (Bornkessel et al., 2006; Grewe et al., 2006), and syntactic complexity (Lee \& Newman, 2010; Newman et al., 2009). A region which lies more anterior to BA 44, BA 45, has been found to be affected by manipulations of plausibility and world knowledge (Friederici et al., 2003; Hagoort et al., 2004) and thematic role assignment (Newman et al., 2003). Because the LIFG has been shown to be so intimately involved in sentence level processing, both syntax and semantics, it was the primary region of interest in this study.

\section{Methods}

Participants. A total of 22 individuals (mean age $=23.6, \mathrm{SD}=$ 3.16; 8 male) from the Indiana University community participated in the study. All participants were required to have English as their native language and be right handed as measured by the Edinburgh handedness inventory. One participant was excluded from analysis due to distortions observed in their imaging data. All participants gave written informed consent approved by the IRB committee of Indiana University prior to their participation.

Materials and Procedures. Before sentence stimuli were constructed a survey was conducted in which participants rated the 
semantic relatedness of noun pairs using a scale of 1-4. The survey was completed by 305 individuals. This information was then used to generate the 125 sentences presented in this study.

The 125 sentences were divided into the two conditions of interest (morphosyntactic violations in semantically related and unrelated sentences) plus filler conditions; fillers were either grammatically correct sentences or sentences with a well-formedness violation. The study was a one factor design with semantic relatedness (semantically related versus unrelated nouns) as a within participant variable. There were 20 sentences in each of these two conditions. Example stimuli include:

Related Nouns

Unrelated Nouns
The bellman serve the traveler and carried the bags to the room.

The boy find the mayor and pokes the mouse with a stick.

As demonstrated in the example stimuli, the unrelated sentences, by virtue of the sentence constituents not being related, are more implausible compared to the related sentences. However, these sentences have been and are currently being used in research. As such, it is of interest to determine how they differ from the more natural sentences that we encounter every day.

A second variable was also introduced, the location of the violation. The violation was either located at the first or second verb. This was done to eliminate the development of a strategy in which participants only focused on one of the two verbs. Once the sentence stimuli were generated to ensure that they were either semantic related or unrelated, another survey was performed in which 32 undergraduates took part. The survey was made up of noun pairs taken from the sentences and participants rated them on a scale 
of 1-4. The results indicated that there was a significant difference between the related and unrelated condition $(\mathrm{p}<0.001)$. The sentence conditions were also equated for the total length of the sentences, animacy of the nouns and frequency of each word.

Participants underwent a training session prior to scanning. During the training session a description of the task was provided and practice trials were completed. Sentences were presented all at once on a screen for 6 sec. Participants responded by pressing a button with the left index finger if the sentence was unacceptable and with their right index finger if it was acceptable. They were told that acceptability judgments should be based on both the semantics and the grammar of the sentences. A slow event-related design was used; therefore, a 12 second rest followed each sentence. The stimuli were divided into 5, 8.75 minute runs. Each run contained 25 sentences and 3, 30 second fixation periods (one at the beginning, middle and end of the run) that served as a common baseline.

fMRI acquisition and analysis. The images were acquired on a 3T Siemens TRIO scanner with an 8-channel radio frequency coil located in the Imaging Research Facility at Indiana University. The functional images were acquired in $185 \mathrm{~mm}$ thick oblique axial slices using the following parameters: $\mathrm{TR}=1000 \mathrm{msec}, \mathrm{TE}=25 \mathrm{msec}$, flip angle $=60^{\circ}$, voxel size $=3.125 \mathrm{~mm} \times 3.125 \mathrm{~mm} \times 5 \mathrm{~mm}$ with a $1 \mathrm{~mm}$ gap.

The data were analyzed using statistical parametric mapping (SPM5 from the Wellcome Department of Cognitive Neurology, London). Standard preprocessing procedures were employed (see Newman et al., 2009). At the individual level, statistical analysis was performed on each participant's data by using the general linear model and Gaussian random field theory as implemented in SPM5. Each event (trial) was convolved with a canonical hemodynamic response function and entered as regressors in the model. 
For the random effects analysis on group data, one-sample t-tests were performed on contrast images to examine main effects (related versus unrelated; each condition vs. fixation). The correction for multiple comparisons for the a priori predicted activation in the left IFG was performed with the use of an uncorrected $p$ value of 0.005 and a cluster size threshold of 20; this corresponds to a per-voxel false-positive probability of less than 0.000001 (Forman et al., 1995), and was used on the related versus unrelated contrast. This method has been utilized previously (Knutson et al., 2004, 2006; Wood et al., 2003; Konishi et al., 1998; Poldrack et al., 1999). In addition, the activated clusters within the IFG were subjected to small volume correction. For the whole-brain analyses, correction for multiple comparisons was carried out using the false discovery rate (FDR) approach (Benjamini \& Yekutieli, 2001; Yekutieli \& Benjamini, 1999) for the condition versus fixation contrasts.

\section{Results}

Predefined ROI analysis. A differential response to semantic relatedness was predicted for the LIFG. Three separate clusters of activation were observed in this a priori ROI (see Table 1 and Figure 1). They included BA $44,46 / 45$, and the posterior part of the inferior frontal sulcus (IFS) - BA 9/44.

Table 1: Predefined Inferior Frontal Gyral Regions

\begin{tabular}{|c|c|c|c|}
\hline Region & $\begin{array}{l}\text { Cluster } \\
\text { size }\end{array}$ & $\mathrm{p}_{\text {FWE-corr }}$ & $\begin{array}{l}\text { Coordinates } \\
\mathrm{x}, \mathrm{y}, \mathrm{z}\end{array}$ \\
\hline
\end{tabular}




\begin{tabular}{ll|lll}
\hline \multicolumn{5}{c}{ Related $>$ Unrelated } \\
\hline Inferior Frontal Gyrus & BA 46/45 & 304 & 0.005 & $-44,50,8$ \\
Inferior Frontal Gyrus & BA 44 & 66 & 0.007 & $-60,10,16$ \\
Inferior Frontal Gyrus & BA 44/9 & 40 & 0.018 & $-54,18,34$ \\
\hline
\end{tabular}

Related

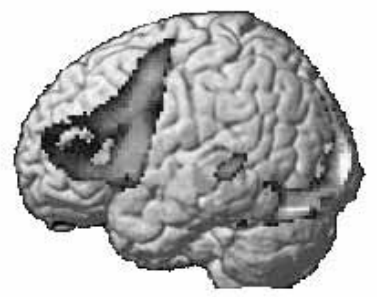

\section{Unrelated}

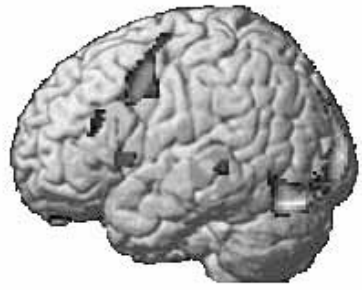

Related - Unrelated

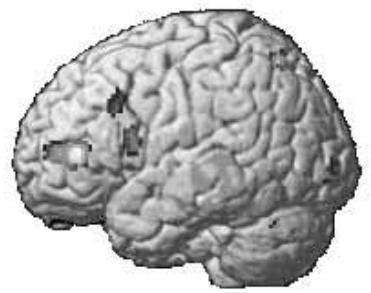

Figure 1. The figure depicts the activation contrasts maps for the related (top left) and unrelated (top right) conditions versus fixation (FDR correction, $\mathrm{p}<$ 0.05). The bottom image is the related minus unrelated contrast (uncorrected, $\mathrm{p}<0.005)$. As shown, there are three clusters within the a priori defined region of interest (IFG).

Whole brain analysis. Whole brain analysis was also performed in which the related and unrelated conditions were compared to a fixation baseline. As shown, there was greater activation for the related compared to the unrelated condition in the commonly activated 
regions. These common regions included the LIFG, precentral sulcus, thalamus and occipital regions (see Table 2 and Figure 1).

Table 2: Whole Brain Analysis (FDR corrected, $\mathrm{p}<0.05$ )

\begin{tabular}{|c|c|c|c|c|}
\hline Region & & $\begin{array}{l}\text { Cluster } \\
\text { size }\end{array}$ & t-value & $\begin{array}{l}\text { Coordinates } \\
\mathrm{x}, \mathrm{y}, \mathrm{z}\end{array}$ \\
\hline \multicolumn{5}{|c|}{ Related $>$ Fixation } \\
\hline Occipital Cortex & BA 18 & 9173 & 9.73 & $-14,-102,2$ \\
\hline Left Hippocampus & & 5158 & 6.17 & $-28,-28,-2$ \\
\hline Left Superior Frontal & BA 6 & 261 & 4.41 & $-4,14,52$ \\
\hline Left Middle Temporal & BA 21 & 199 & 3.65 & $-62,-42,2$ \\
\hline Right Middle Frontal & BA 46 & 191 & 3.89 & $46,44,24$ \\
\hline Left Superior Parietal & BA 7 & 37 & 3.16 & $-26,-58,42$ \\
\hline Right Thalamus & & 33 & 3.82 & $28,-26,0$ \\
\hline Left Cerebellum & & 16 & 3.46 & $-36,-44,-28$ \\
\hline \multicolumn{5}{|c|}{ Unrelated $>$ Fixation } \\
\hline Occipital & BA 17 & 7089 & 8328 & $-8,-90,-6$ \\
\hline Basal Ganglia & & 709 & 5.17 & $-26,-4,26$ \\
\hline Anterior Insula & BA 13 & 85 & 3.99 & $-52,12,4$ \\
\hline Parahippocampus & & 76 & 4.87 & $-32,-28,-4$ \\
\hline Left Temporal & BA 21 & 39 & 3.46 & $-50,-38,-2$ \\
\hline Inferior Frontal & $\begin{array}{l}\text { BA } \\
46 / 45\end{array}$ & 28 & 3.66 & $-52,26,24$ \\
\hline
\end{tabular}

In addition to examining the two anomaly conditions, the effect of relatedness was also examined for the filler, non-anomalous sentences. This was performed to test the hypothesis mentioned in the introduction - in the absence of an anomaly, related sentences will be processed more efficiently than unrelated. The imaging results support this hypothesis. Using an uncorrected threshold 
of $\mathrm{p}<0.001$ and an extent of 20 voxels, unlike for the anomalous sentences the related non-anomalous sentences failed to elicit more activation than the unrelated sentences, even at a lower threshold of $\mathrm{p}<0.005$ and a 0 extent threshold. However, the unrelated sentences did elicit increased activation in the bilateral superior frontal cortex, parahippocampus and the anterior cingulate cortex (see Table 3). No traditional language processing regions (i.e., LIFG) revealed effects.

Table 3: Filler sentence activation (uncorrected $p<0.001$, extent 20)

\begin{tabular}{ll|lll}
\hline Region & $\begin{array}{l}\text { Cluster } \\
\text { size }\end{array}$ & $\mathrm{t}$-value & $\begin{array}{l}\text { Coordinates } \\
\mathrm{x}, \mathrm{y}, \mathrm{z}\end{array}$ \\
\hline \multicolumn{5}{c}{ Unrelated $>$ Related } \\
\hline Parahippocampus & & 122 & 4.32 & $-24,-40,-12$ \\
Left Superior Frontal & 9 & 98 & 4.62 & $-24,44,28$ \\
Anterior Cingulate & $24 / 32$ & 44 & 4.88 & $-4,22,12$ \\
Right Superior Frontal & 9 & 28 & 3.96 & $24,42,30$ \\
\hline
\end{tabular}

\section{Ancillary behavioral study}

A total of 45 students (mean age $=19.5, \mathrm{SD}=1.25$ ) from the Indiana University community participated in the study. All participants were required to have English as their native language. All participants gave written informed consent approved by the IRB committee of Indiana University prior to their participation.

A self-paced reading paradigm was employed in which sentences, the same sentences used in the fMRI study, were presented one word at a time in the center of the screen. Participants pressed the space bar to move to the next word, allowing for the collection of word reading times. Following each sentence participants indicated whether the sentence was acceptable. Participants pressed the "A" key with their 
left index finger to denote "unacceptable" and the "L" with their right index figure to denote "acceptable;" in other words, right hand was "right" (acceptable) and left hand was wrong (unacceptable).

One of the main findings in this behavioral study was that for the anomalous sentences the unrelated condition elicited higher accuracy scores than did the related condition $\left[\mathrm{F}_{1}(1,44)=26.45\right.$, $\left.\mathrm{MSE}=1.166, \mathrm{p}<0.0001 ; \mathrm{F}_{2}(1,38)=16.66, \mathrm{MSE}=0.251, \mathrm{p}<0.0005\right]$, see Figure 2. Also, there was no effect of anomaly position - the first or second verb in the sentence $-\left[\mathrm{F}_{1}=0, \mathrm{MSE}=0.0 ; \mathrm{F}_{2}=0, \mathrm{MSE}=0.0\right]$.

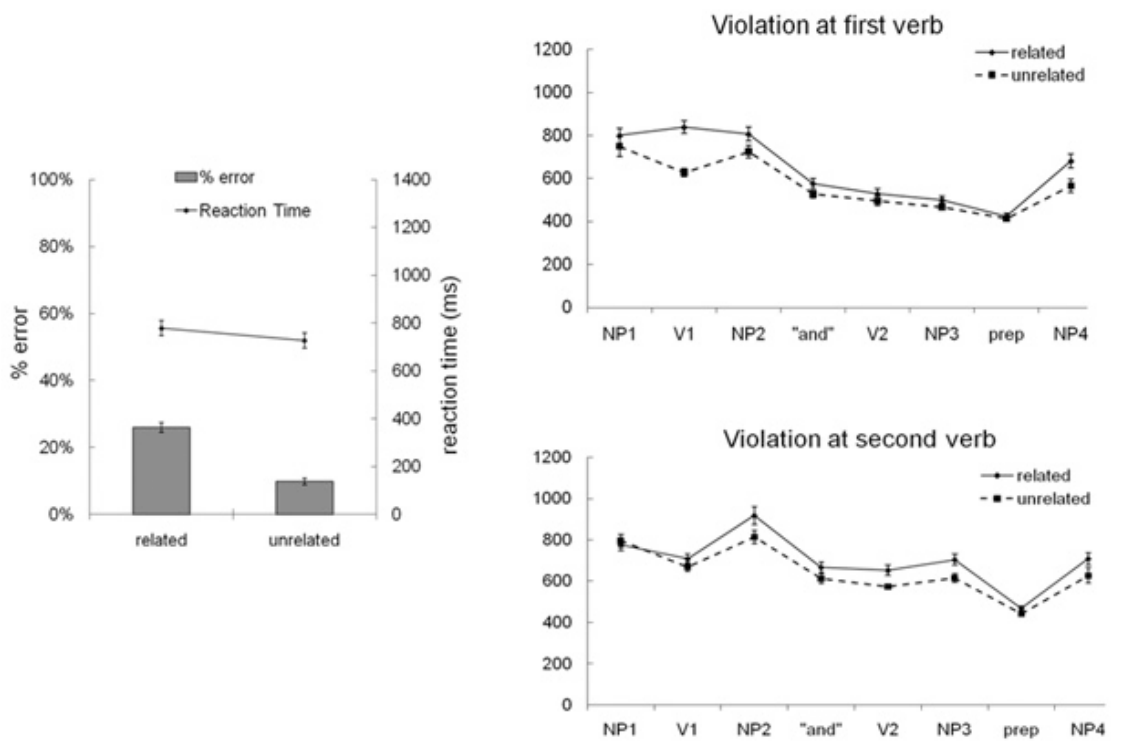

Figure 2. Behavioral results show (on the left) the significant effect of relatedness on the error rate $[\mathrm{F}(1,44)=26.45, \mathrm{MSE}=1.166, \mathrm{p}<0.0001]$ with the related condition eliciting a larger number of errors. The line graphs on the right are word reading times for the conjoined active sentences. The violation was positioned either at the first or the second verb. As shown, the verb that contained the morphosyntactic violation showed slower processing times in the related condition. 
The RT analysis was performed only on correct trials. No reaction time differences were observed when comparing related to unrelated sentences $\left[\mathrm{F}_{1}(1,44)=3.26, \mathrm{MSE}=574748, \mathrm{p}=0.08 ; \quad \mathrm{F}_{2}(1,39)=1.02\right.$, MSE $=24090, p>0.3]$ or when comparing the location of the anomaly $\left[\mathrm{F}_{1}=0.33, \mathrm{MSE}=40508 ; \mathrm{F}_{2}=0.54, \mathrm{MSE}=13364\right]$.

Finally word reading times were analyzed, only for correct responses (see Supplementary Material). The second major finding here was that the word reading times at and following the anomaly (the wrong tense verb) was longer for the related compared to the unrelated sentences (see Figure 2) suggesting that additional processing is necessary to generate the correct response if the sentence constituents are semantically related.

Like for the imaging data, an analysis of the filler, non-anomalous sentences was also performed and like for the imaging data reverse trends were observed. Here, the unrelated condition elicited longer RTs for the correctly answered trials [unrelated $M=734.4 \mathrm{~ms}$; related $\left.\mathrm{M}=697.6 ; \mathrm{F}_{1}(1,44)=8.03, \mathrm{p}<0.008\right]$. The error data also revealed a significant effect with the unrelated condition eliciting more errors than the related condition $\left[\mathrm{F}_{1}(1,44)=74.64, \mathrm{p}<0.001\right]$. Finally, the mean word reading times for the correctly answered trials were longer for the unrelated condition $\left[\mathrm{F}_{1}(1,44)=13.4, \mathrm{p}<0.001\right]$.

\section{General discussion}

The primary aim of the current study was to determine whether semantics has a significant impact on syntactic analysis. The results presented here suggest that it does. Three subregions of the left inferior frontal gyrus were found to show a differential response to the semantic relatedness manipulation during anomaly detection. These three regions have been implicated in different cognitive processes, 
including syntactic, memory and cognitive control processes. In addition, the results from the behavioral study showed that the related anomalous sentences elicited more errors, and had a longer reaction and reading times. Finally, when the non-anomalous sentences were examined the reverse trend was observed, the unrelated sentences elicited greater activation and longer reaction and reading times than did the related sentences, suggesting that the effect of relatedness is linked to the introduction of an anomaly.

One of the three regions within the LIFG that revealed a significant effect of relatedness when processing the anomalous sentences was BA 44. There are a number of studies that have implicated BA 44 in syntactic processing (Friederici et al. 2003; Newman et al. 2003) - particularly when the syntactic complexity of the sentence structure is manipulated - as well as in the processing of prominence information (Bornkessel \& Schlesewsky, 2006; Grewe et al., 2006). The observation of an effect of relatedness in this region for the anomalous sentences suggests that relatedness is affecting some aspect of syntactic processing other than complexity, given that there is no syntactic complexity manipulation here. Finding a null effect of relatedness in the region for the filler, non-anomalous sentences support this hypothesis. It is important to note that BA 44 has been implicated in more than syntactic processing. A number of neuroimaging studies have found activation of the region during inflectional morphology tasks (Sahin et al., 2006; Dasai et al., 2006; Tyler et al., 2004; 2005). For example, Tyler and colleagues (2004) found that the LIFG, including BA44, was implicated in the processing inflected forms, particularly regular forms, even when those forms were non-words. Sahin et al. (2006) also found similar involvement of the LIFG when comparing inflected and non-inflected nouns and verbs in a covert sentence completion task. However, the role of the 
LIFG in inflectional morphology is not clear. One hypothesis that explains the involvement of BA 44 in both syntactic complexity and inflection morphology is that these two language functions rely on overlapping processes, namely rule-based, combinatorial processing, and this is the function of BA 44. Therefore, it may be that in the current study the role of BA 44 is not syntactic as much as it is morphological.

The more anterior cluster of activation within LIFG that revealed an effect of relatedness for the anomalous sentences was BA 45/46. This region has been linked to the retrieval of information from declarative memory stores (Anderson et al., 2008) and it overlaps with regions that have been implicated in studies of semantic retrieval (Wagner et al., 2000; Gold et al., 2006; Badre et al., 2005). For example, in a study conducted by Badre and colleagues it was suggested that the region is involved in "the selection of task-relevant representations from retrieved alternatives" (p. 914). Also, Gold and Buckner (2002) found that the lateral inferior prefrontal cortex was involved in controlled retrieval from memory for both semantic and non-semantic information. In that study a semantic task (deciding whether a visually presented word is abstract or concrete) and a non-semantic task (deciding whether visually presented words and pseudowords contain a long or short vowel) requiring controlled retrieval of information from memory were found to elicit the involvement of the lateral prefrontal cortex. The prediction regarding the involvement of this region may have been that it would be more involved for the unrelated condition due to the sentence constituents not being related and possibly not priming the retrieval of each other the way it may be for the related condition. However, here the reverse was observed for the anomalous sentences and there was no difference in the activation of the region for the non-anomalous 
sentences. There is an alternative explanation. The differential involvement of BA 45/46 may be related to the anomaly itself in that declarative memory must be accessed to aid in the recollection of the subject-verb agreement rules in order to respond correctly. Further studies are required to disentangle the role of BA 45/46 here.

The third region of the LIFG that revealed a significant effect of relatedness for the anomalous sentences is the region at the junction of the inferior frontal sulcus and the inferior precentral sulcus the inferior frontal junction (IFJ). This region has been implicated in cognitive control (Brass \& von Cramon, 2002, 2004; Bunge et al., 2003; Derrfuss et al., 2004; Konishi et al., 2001). In a study conducted by Derrfus and colleagues (2004) the IFJ was found to be involved in a task-switching paradigm, in the Stroop task, and in the n-back task. There they suggested that the region was involved in the "representation of task rules." The "good enough" hypothesis of language processing purports that the language system often times generates shallow or superficial representations, and most often the inaccuracies that result from the use of this heuristics are consistent with the plausibility of events in the real world (Christianson et al., 2003; Ferreira, Christianson, \& Hollingworth, 2001). This hypothesis fits the data presented here in that the semantic relatedness of the sentence constitutes makes the sentence more plausible in the real world and allows for a more shallow representation to be generated. This shallow representation may be expected to make the detection of a morphosyntactic violation more difficult. This explanation fits the current data in that for the related anomalous sentences may require greater control to over-ride the proponent response that is generated by the semantics of the sentence. Again, because the sentence describes events that are plausible and familiar it is more difficult to pick up on the morphosyntactic violation. Control processes, such 
as remembering that the task is not comprehension but to determine whether there is an error, will be recruited more for the anomalous related sentences. In the case of the unrelated condition, the events described in the sentences are not as plausible and the comprehension not as easy or fast, so there is less conflict and, therefore, less control necessary to inhibit the comprehension processes.

\section{Conclusions}

Under typical conditions, a comprehender's task is to comprehend, understand the message, not to "prove the accuracy or detailed nature of their understanding" (Ferreira \& Patson, 2007, p.71). Therefore, the use of fast, efficient heuristics should allow for facilitation of processing under certain task conditions and when processing non-anomalous sentences. Evidence to support this idea was found here. The filler, non-anomalous sentences in the behavioral study were read faster and had a faster RT. The reverse of what was found for the anomalous sentences. Also, the unrelated fillers elicited increased activation compared to the related sentences in the parahippocampus and bilateral superior frontal cortex. No region revealed increased activation for the related condition for the filler sentences. All of this suggests that "good enough" processing may be taking place but this "good enough" processing is not good enough for all types of language tasks.

\section{Note}

1. Acknowledgements: This research was supported by a National Institute of Health grant (R03 HD051579-01).

Address correspondence to:

Sharlene D. Newman

Department of Psychological and Brain Sciences 
Indiana University

1101 E. 10th St

Bloomington, IN 47405

Tel: 812-856-0839

\section{References}

Anderson, J.R., Fincham, J.M., Qin, Y., \& Stocco, A. (2008). A central circuit of the mind. Trends in Cognitive Science, 12: 136-143.

Badre, D., Poldrach, R.A., Pare-Blagoev, E.J., Insler, R.Z., Wagner, A.D. (2005) Dissociable controlled retrieval and generalized selection mechanisms in ventrolateral prefrontal cortex. Neuron, 47: 907-918

Benjamini Y, Yekutieli D. (2001). The control of false discovery rate in multiple testing under dependency. Annals of Statistics, 29:1165-1188.

Bornkessel, I., Schlesewsky, M. (2006). The Extended Argument Dependency Model: A neurocognitive approach to sentence comprehension across languages. Psychological Review 113: 787-821.

Bornkessel-Schlesewsky, I. \& Schlesewsky, M. (2008). An alternative perspective on "semantic P600" effects in language comprehension. Brain Research Reviews, 59:55-73.

Brass, M., von Cramon, D.Y. (2002). The role of the frontal cortex in task preparation. Cerebral Cortex 12, 908- 914.

Brass, M., von Cramon, D.Y. (2004). Decomposing components of task preparation with functional MRI. Journal of Cognitive Neuroscience, 16: 609-620.

Bunge, S.A., Kahn, I., Wallis, J., Miller, E., Wagner, A. (2003). Neural circuits subserving the retrieval and maintenance of abstract rules. J. Neurophysiol. 90, 3419-3428.

Christianson, K., Hollingworth, A., Halliwell, J. \& Ferreira, F. (2001). Thematic roles assigned along the garden path linger. Cognitive Psychology, 42: 368-407.

Dasai, R., Conant, L.L., Waldron, E., \& Binder, J.R. (2006). fMRI of past tense processing: the effects of phonological complexity and task difficulty. Journal of Cognitive Neuroscience, 18: 278-297. 
Derrfuss, J., Brass, M., \& von Cramon, D. Y. (2004). Cognitive control in the posterior frontolateral cortex: evidence from common activations in task coordination, interference control, and working memory. NeuroImage, 23:604-612.

Ferreira, F., Patson, N.D. (2007). The 'good enough' approach to language comprehension. Language and Linguistics Compass, 1: 71-83.

Ferreira, F., V. Ferraro, and K. G. D. Bailey. 2002. Good-enough representations in language comprehension. Current Directions in Psychological Science, 11:11-15.

Ferreira, F., Christianson, K. \& Hollingworth, A.(2001). Misinterpretations of garden-path sentences: implications for models of reanalysis. Journal of Psycholinguistic Research, 30:3-20.

Forman SD, Cohen JD, Fitzgerald M, Eddy WF, Mintun MA, Noll DC. (1995). Improved assessment of significant activation in functional magnetic resonance imaging (fMRI): Use of a cluster-size threshold. Magnetic Resonance in Medicine, 33:636-647.

Friederici AD, Ruschemeyer SA, Hahne A, Fiebach CJ. (2003) The Role of left inferior frontal and superior temporal cortex in sentence comprehension: localizing syntactic and semantic processes. Cerebral Cortex. 13:170-177.

Gold, B.T., \& Buckner, R.L., (2002). Common Prefrontal Regions Coactivate with Dissociable Posterior Regions during Controlled Semantic and Phonological Tasks. Neuron, 35: 803-812.

Gold, B.T., Balota, D.A., Jones, S.J., Powell, D.K., Smith, C.D., \& Andersen, A.H. (2006) Dissociation of automatic and strategic lexical semantics: fMRI evidence for differing roles of multiple frontotemporal regions. J. Neurosci. 26:6523-6532

Grewe, T., Bornkessel, I., Zysset, S., Wiese, R., von Vramon, D.Y., Schlesewsky, M. (2006). Linguistic prominence and Broca's area: the influence of animacy as a linearization principle. NeuroImage, 32:13951402.

Hagoort, P., C. Brown and J. Groothusen, (1993) The syntactic positive shift (SPS) as an ERP measure of syntactic processing, Language and Cognitive Processes, 8:439-483. 
Hagoort, P., Hald, L., Bastiaansen, M., Petersson, K.M. (2004). Integration of word meaning and world knowledge in language comprehension. Science, 304: 438-441.

Hoeks, J.C.J., Stowe, L.A., Doedens, G. (2004). Seeing words in context: the interaction of lexical and sentence level information during reading. Cognitive Brain Research 19: 59-73.

Kim, A., Osterhout, L. (2005). The independence of combinatory semantic processing: evidence from event-related potentials. Journal of Memory and Language, 52: 205-225.

Knutson KM, Wood JN, Grafman J. (2004). Brain Activation in Processing Temporal Sequence: an fMRI Study. NeuroImage, 23:1299-1307.

Knutson, KM, Wood, JN, Spampinato \& Grafman, J. (2006). Politics on the Brain: An fMRI Investigation. Social Neuroscience, 1: 25-40.

Konishi S, Nakajima K, Uchida I, Sekihara K, Miyashita Y. (1998). No-go dominant brain activity in human inferior prefrontal cortex revealed by functional magnetic resonance imaging. European Journal of Neuroscience, 10:1209-1213.

Konishi, S., Donaldson, D.I., Buckner, R.L., 2001. Transient activation during block transition. NeuroImage 13, 364-374.

Kuperberg, G.R., Kreher, D.A., Blais, K., et al. (2005). Semantic influences on syntactic processing: evidence from event-related potentials. Journal of Cognitive Neuroscience, Suppl. Abstract.

Lee, D., Newman, S.D. (2010). The effect of presentation paradigm on syntactic processing: An event-related fMRI study. Human Brain Mapping, 31:123-132.

Newman SD, Just MA, Keller TA, Roth J, Carpenter PA (2003) Differential effects of syntactic and semantic processing on the subregions of Broca's area. Cognitive Brain Research, 16:297-307.

Newman, S.D., Ikuta, T. \& Burns, T. (2010). The effect of the semantic relatedness on sentence comprehension: an fMRI study. Brain and Language, 113: 51-58

Newman, S.D., Lee, D., Ratliff, K. (2009). How much does the comprehension probe interact with on-line syntactic processing? Human Brain Mapping, 30: 2499-2511. 
Ni,W., Constable, R.T., Mencl,W.E., Pugh, K.R., Fulbright, R.K., Shaywitz, S.E., Shaywitz, B.A., Gore, J.C., \& Shankweiler, D. (2000). An eventrelated neuroimaging study distinguishing form and content in sentence processing. Journal of Cognitive Neuroscience, 12: 120-133.

Poldrack RA, Wagner AD, Prull MW, Desmond JE, Glover GH, Gabrieli JDE. (1999). Functional specialization for semantic and phonological processing in the left inferior prefrontal cortex. NeuroImage, 10:15-35.

Sahin, N.T., S. Pinker and E. Halgren, (2006) Abstract grammatical processing of nouns and verbs in Broca's area: Evidence from fMRI, Cortex, 42:540-562.

Sanford, A. J., and P. Sturt. 2002. Depth of processing in language comprehension: not noticing the evidence. Trends in Cognitive Sciences, 6:382-6.

Tyler, L. K., Stamatakis, E. A., Bright, P., Acres, K., Abdallah, S., Rodd, J. M. \& Moss, H. E. (2004) Processing objects at different levels of specificity. Journal of Cognitive Neuroscience, 16:351-362.

Tyler, L. K., Stamatakis, E. A., Post, B., Randall, B. \& Marslen-Wilson, W. D. (2005) Temporal and frontal systems in speech comprehension: an fMRI study of past tense processing. Neuropsychologia, 43:1963-1974.

Wagner AD, Koutstaal W, Maril A, Schacter DL, Buckner RL (2000): Taskspecific repetition priming in left inferior prefrontal cortex. Cerebral Cortex, 10:1176-1184.

Wason, P., \& Reich, S.S. (1979). A verbal illusion. Quarterly Journal of Experimental Psychology, 31, 591-597.

Wood JN, Romero SG, Makale M, Grafman J. (2003). Category-specific representations of social and nonsocial knowledge in the human prefrontal cortex. Journal of Cognitive Neuroscience, 15:236-248.

Yekutieli D, Benjamini Y. (1999). Resampling-based false discovery rate controlling multiple test procedures for correlated test statistics. Journal of Statistical Planning and Inference, 82:171-196.

[Received in 19/08/2011. Approved in 13/02/2012] 
